\title{
Die Erfolge der Salvarsanbehandlung bei Nervenkrankheiten.)
}

\author{
Von \\ Privatdozent Dr. Emil Mattausehek, \\ k. u. k. Stabsarzt.
}

(Aus der neurologisch-psychiatrischen Abteilung des k. u. k. Garnisonsspitals Nr. 1 in Wien.

(Eingegangen am 22. März 1911.)

Mehr als Jahresfrist ist verflossen, seitdem Alt über die ersten Behandlungsversuche und Erfolge berichtet hat, die er gemeinsam mit Hop pe und Schreiber teils an neurologisch-psychiatrischem Materiale, teils an Fällen mit florider Syphilis durch Anwendung des Ehrlichschen Dioxydiamidoarsenobenzols beobachten konnte.

Eine kaum mehr zu überblickende internationale Literatur gibt Zeugnis über eine ungeheure, mit größter Begeisterung geleistete Arbeit, welche die Feststellung der Wirksamkeit des Salvarsans in der Behandlung der Syphilis und ihrer Folgekrankheiten zum Ziele hatte. Nichtsdestoweniger lassen sich auch heute noch trotz des bisher gesammelten überaus reichen klinischen Beweismateriales eine Reihe von Fragen noch nicht definitiv beantworten. So sind die Akten über die Möglichkeit von Abortivkuren im Sinne der Therapia magna sterilisans noch immer nicht ganz geschlossen, es ist die Frage hinsichtlich Dauerresultaten nicht in letzter Linie infolge der recht ungleich wirksamen zahlreichen Methoden noch ungeklärt und überhaupt verfrüht, die Erwägung einer eventuellen Verhütung der Nachkrankheiten eine theoretisch spekulative.

Immerhin erscheint mir mit Rücksicht auf die Häufigkeit syphilitischer und syphilogener Erkrankungen des Nervensystems der Versuch gerechtfertigt, zusammenfassẻnd über die bisherigen Erfahrungen in der Behandlung derartiger Erkrankungen zu berichten.

Ich muß absehen von einer speziellen Anführung der zahlreichen Einzelbeobachtungen und will auch nicht die bereits vorliegenden Arbeiten, welchen größere Beobachtungsreihen zugrunde liegen, gesondert vorbringen. Ich will nur versuchen, aus dem in verwertbarer

1) Vortrag, gehalten am 14. März 1911 im Verein für Psychiatrie und Neurologie in Wien.

Z. f. d. g. Neur. u. Pgych. O. IV. 
Form bisher publizierten Materiale und an der Hand meiner eigenen Beobachtungen die wesentlichsten Ergebnisse herauszugreifen.

Im allgemeinen haben die Erfahrungen gezeigt, daß die luetisch bedingten Erkrankungen des Nervensystems ein dankbares Gebiet der Salvarsantherapie darstellen.

Betont mag an dieser Stelle gleich werden, daß die ursprünglich gehegten Befürchtungen einer Gefahr für den Sehnerven sich nicht bestätigt haben, und daß eine direkt toxische Wirkung des Salvarsans an sich auf die Hirnnerven (Fälle von Finger, Rille, Spiethoff, Stern, Beck, Matzenauer) keineswegs bewiesen ist. Hirnnervenstöru ngen nach Salvarsanbehandlung kommen mit Ausnahme der Frühperiode der Syphilis kaum zur Beobachtung. Sie scheinen eine durch ihre Art und den Zeitpunkt des Auftretens besondere Form der Syphilisrezidive darzustellen, welche wie alle anderen luetischen Hirnnervenaffektionen mit bestem Erfolge einer Behandlung mit Salvarsan oder $\mathrm{Hg}$ zugänglich sind. Utber solche Fälle verdanken wir eine reiche und belehrende Kasuistik Jgersheimer, Grósz, Schanz, Hirsch, Favento, Frenkel, Géronne und Guttmann.

Ebenso gehören Schädigungen der peripheren Nerven, sofern sie nicht durch Fehler der Technik und örtliche Entzündungserscheinungen bedingt sind, zu den Ausnahmen. - Vorwegnehmen will ich, daß wir unsere Erwartungen überhaupt nicht zu hoch spannen, daß wir bei den vielen Spätformen infolge der tiefergreifenden Prozesse nur insoweit Erfolge verlangen dürfen, als re parable Störungen vorliegen.

Es liegt in der Natur der Sache und der spezifisch bakteriziden bzw. spirillotropen Wirkung des Präparates, daß das Hauptanwend ungsgebiet desselben in erster Linie in allen jenen Erkrankungsformen liegt, welche direkt durch Spirochäten verursacht sind, d. h. in allen Fällen, gegen welche wir bisher $\mathrm{Hg}$ und Jod mit Erfolg angewendet oder wenigstens als angezeigt gehalten haben.

Die Durchsicht der Literatur ergibt bezüglich derartiger Fälle fast durchaus günstige Urteile. So bezeichnet Alt die Gehirnsyphilis als das überzeugendste und dankbarste Objekt der Salvarsanbehandlung.

Ähnlich günstig äußern sich über ihre Erfahrungen bei den verschiedenen Formen der Gehirn- und Rückenmarkssyphilis fast alle späteren Autoren. Ich zitiere nur Bruhns, Duhot, Favento, Frenkel, Jadassohn, Marcus, Marinesco, Matzenauer, Neisser, Treupel, Willige u. a.

Im besonderen geben selbstverständlich alle Fälle um so mehr Aussicht auf vollkommene Erfolge, je frischer die gesetzten Schädigungen sind, während Symptome und Erkrankungsformen, welche auf anato- 
misch irreparablen, degenerativen Zerstörungen beruhen, nicht beeinflußt werden. Diese Tatsachen sind zu bekannt, sie decken sich mit unseren Erfahrungen mit der $\mathrm{Hg}$ - und Jodtherapie, und erübrigt es sich, darauf näher einzugehen.

Der Hauptvorzug des Salvarsans liegt aber meines Erachtens nicht in der Einfachbeit und Kürze der Kur, sondern darin, daß das Salvarsan gerade in alten Fällen und auch dort vorzügliche Erfolge zu geben imstande ist, wo $\mathrm{Hg}$ und Jod versagt haben oder unanwendbar sind. Ferner - und das ist der für die neurologische Praxis wichtigste Punkt - erweist sich das Salvarsan dem $\mathrm{Hg}$ in der Raschheit des Eintrittes der Heilwirkung weit überlegen (vgl. die Beobachtungen von Gennerich, Matzenauer, Michaelis, Plehn usw.). Der Umstand, daß die Wirkung dort, wo sie überhaupt eintritt, sich rasch, oft verblüffend schnell zeigt, hat dazu geführt, die syphilitischen Erkrankungen des Gehirns und Rückenmarks als besonderes Indikationsgebiet zu bezeichnen. In diesem Sinne haben sich mit allem Nachdruck Bruhns, Fraenkel, Friedländer, Géronne, Neisser, Werther und jüngst wieder Marinesco ausgesprochen. Es liegen in dieser Richtung zahlreiche Beobachtungen vor, welche lebens- und funktionsrettende Erfolge darstellen, wie sie durch die bisherige Therapie nicht erreicht werden können. Hierher gehören Fälle von gummöser Meningitis mit heftigen Kopfschmerzen, Neuritis opt., Hirnnervenstörungen, Fälle von spinaler Lues, Meningomyelitiden, frische luetische Hemiplegien, luetische Epilepsien u. dgl., bei welchen die rech tzeitig ein setze nde Salvarsantherapie durch die fast momentane Befreiung von Schmerzen, die Wiederherstellung der Funktion, die Verhütung der Entwicklung degenerativer Veränderungen die glänzendsten und befriedigendsten Heileffekte erzielen kann, die überhaupt erreichbar sind. In Fällen alter Meningomyelitis (Nonne, Oppenheim), bei syphilitischen Spinalparalysen (Oppenheim, Weintraud), in alten Fällen spinaler und cerebraler Lues werden wir nicht viel erwarten können und uns mit Besserungen und Beseitigung von Einzelsymptomen, Stillständen begnügen müssen. Daß aber auch in spätluetischen Fällen, bei luetischen Hemiplegien, bei endarteritischen Prozessen noch ganz schöne Resultate zu erzielen sind, haben die Beobachtungen von Brasch, Duh ot, Frenkel, Gennerich, Salmon, Schlesinger u. a. gezeigt. Nicht unerwähnt will ich lassen, daß nach einzelnen Beobachtungen Pupillendifferenzen und Unregelmäßigkeiten, Reflexstörungen der Pupillen zum Ausgleiche kamen (Hügel-Ruete, Marcus, Plehn).

Besonders gute Erfolge gibt die Behandlung von Fällen latenter und tertiärer Lues, bei welchen mehr oder weniger schwere neurasthenische Erscheinungen vorliegen, direkt luetische Symptome 
mit Ausnahme eines positiven Wassermanns fehlen. Auf diese Fälle hat in letzter Zeit Fauser speziell hingewiesen. Er hält gerade bei solchen die Salvarsanbehandlung für direkt indiziert, weil seiner Erfahrung nach solche syphilitische Neurasthenien nicht selten Vorläufer schwer destruktiver Prozesse sind.

Diesen günstigen Ergebnissen steht eine nicht unerhebliche Anzahl von Versagern gegenüber. Es unterliegt keinem Zweifel, daß bei einem Teil davon - und zwar gewiß nicht häufiger als beim $\mathrm{Hg}$ das Präparat als solches sich als wirkungslos erweist. Teilweise haben sie ihre Ursache darin, daß bereits irreparable Veränderungen vorlagen, teilweise in der angewendeten Methode.

Daß auch effektiv schädliche Wirkungen beobachtet wurden, ist bekannt. Ich will von vorübergehenden Nebenwirkungen nicht sprechen, die seltenen Neurorezidive und Hirnnervenschäden nur kurz erwähnen. Sie stellen, soweit sie überhaupt mit der Salvarsanbehandlung in kausalem Zusammenhang stehen, doch nur Ausnahmsfälle dar und gehören nahezu ausschließlich der Frühperiode der Syphilis an, die für uns nicht in Betracht kommt.

Soweit es sich um echt syphilitische Nervenkrankheiten handelt, kann ich in der mir zugänglichen Literatur, abgesehen von vereinzelten vorübergehenden Störungen, nur drei Fälle finden, welche bemerkenswert sind. In einem Falle Bonhoeffers trat bei einer schweren spinalen Lues, bei der schon vorher eine in ihrer Intensität schwankende Paraparese und totale Blasenlähmung bestanden hatte, nach der Injektion vollkommene Blasenlähmung und vollkommene Parese des linken Beines auf. Spiethoff beobachtete bei einem Kranken mit latenter sekundärer Lues und Stupor nach der Injektion einen epileptischen Krampfanfall, Eversbusch bei einem Kinde, welches vor 3 Jahren eine Meningitis durchgemacht und eine spastische Parese eines Beines behalten hatte, nach der Behandlung mit Salvarsan sehr heftige epileptische Anfälle.

Aus solchen Fällen und aus der weiteren Erfahrung werden wir zur Vermeidung ähnlicher Mißerfolge noch vieles zu lernen haben, weshalb die Veröffentlichung aller derartigen Fälle dringend wünschenswert ist. Wie bekannt, hat Ehrlich unter anderem bei frischen Hirnblutungen vor der Anwendung des Salvarsans gewarnt. Auf Grund ihrer Beobachtungen haben mehrere Autoren (Gennerich, Werther u. a.) bei frischen luetischen Hemiplegien, bei größeren Hirnherden zur größten Vorsicht gemahnt.

Es sei hier nur auf die Möglichkeit einer schädigenden Wirkung nach Art der Herxheimerschen Reaktion speziell bei mit Hirndruck einhergehenden tumorösen Formen und auf die Gefahr von akuter Hirnschwellung (vgl. Pötzl - Schüller) hingewiesen. 
Auch eine Reihe von Todesfällen nach Salvarsanbehandlung wurde beobachtet.

Dieselben betrafen zum Teil vorgeschrittene oder Anfallsparalytiker (2 Fälle Williges, 1 Fall von Ehlers-Jorgensen, 1 Fall von A. Pick), ferner 2 moribund injizierte Fälle (W. Pick, Gumma pontis; Nonne, arteritische Hirnlues), 1 alte Ponserweichung, 1 Hirnblutungsherd, 1 Tumor, 1 marastische Tabes (Marcus). In einigen Fällen lag der letale Ausgang sicher in der Methode - intravenöse Anwendung von 0,8 Arsenobenzol in saurer Lösung (Schottmüller), konzentrierte, intravenöse Injektion 0,6:20 ccm (Fren kel - Gro u ve n).

Bevor ich die Besprechung der echt syphilitischen Erkrankungen verlasse, will ich in Kürze über meine eigenen Fälle dieser Gruppe berichten. Ich habe mit Doerr gemeinsam im April 1910 die Behandlungsversuche aufgenommen. Die Fälle wurden insofern ausgewählt, als Kranke mit komplizierenden schweren Gefäßerkrankungen, mit Myokarditis, mit vorgeschrittenen degenerativen Erkrankungen des Nervensystems von der Behandlung ausgeschlossen wurden. Dem Zustande der Hirnnerven wurde in allen Fällen vor und nach der Behandlung das größte Augenmerk zugewendet. Die serologische Blutuntersuchung wurde in dem bakteriologischen Laboratorium des Militärsanitätskomitees vorgenommen, die Injektionen bis zur Freigabe des Präparates in demselben Institute jedesmal frisch vorbereitet und von Herren des Laboratoriums durchgeführt ${ }^{1}$ ). Angewendet wurde anfänglich die alkalische Lösung nach der Altschen Methode, später monacide klare Lösungen in einer Flüssigkeitsmenge von ca. $12 \mathrm{ccm}$ (vgl. Matzenauer, Ullmann, Zeissl). Die Injektionen wurden mit wenigen Ausnahmen nicht a mbulatorisch gemacht, durchgehends intramuskulär im oberen äußeren Anteile der Glutäalmuskulatur gesetzt, die Injektionsstellen nicht massiert, mehrere Tage Bettruhe angeordnet. Die behandelten entlassenen Kranken wurden periodisch persönlich kontrolliert, später zu brieflichen Nachrichten über bestimmte Fragepunkte eventuell unter Zuziehung eines Arztes verhalten.

Ich verfüge zurzeit über Beobachtungen von $69 \mathrm{Fällen,} \mathrm{welche}$ sich auf so ziemlich alle in Betracht kommenden Krankheitsformen erstrecken.

Vor allem will ich betonen, daß ich in keine $\mathrm{m}$ Falle eine der Therapie zur Last fallende Hirnnervenstörung, niemals eine Hautnekrose, nie einen Da uerschade n beobachtet habe. Größere Infiltrate, aber ohne jede weitere Störung, kamen bei besonders empfindlichen Patienten oder bei solchen, welche die angeordnete absolute Ruhe nicht eingehalten haben, mehrmals vor. Von Fällen echt syphilitischer

1) Es sei mir gestattet, auch an dieser Stelle den Herren Doz. Dr. Russ und Dr. Moldovan wärmstens zu danken. 
Erkrankung kamen 31 zur Behandlung, davon 6 Fälle latenter Lues mit schwer nervösen Erscheinungen; die übrigen verteilen sich auf die verschiedenen Formen der Lues des Nervensystems (cerebrale, spinale Lues, Meningomyelitis, luetische Hemiplegien, Endarteriitis, Augenmuskellähmungen, Neuralgien usw.).

Von diesen 31 Fällen sind für die Beurteilung des Erfolges nur 27 Fälle verwertbar, da 2 sich der weiteren Untersuchung entzogen, 2 wegen zu kurzer Beobachtungsdauer bzw. Unklarheit des Falles nicht einbezogen werden konnten. 16 von diesen 27 konnten geheilt bzw. so weitgehend gebessert werden, daß sie ihre volle Berufsfähigkeit wieder erlangt haben, 3 wurden dauernd, 4 vorübergehend gebessert, 4 Fälle erfolglos behandelt: ein Fall von Augenmuskellähmung, eine luetische Demenz, ein mehrere Monate alte schwere Hemiplegie, sowie ein im Status epilepticus eingebrachter Fall, welcher fast in moribundem Zustande injiziert wurde.

Von den 16 mit günstigstem Erfolge behandelten Fällen will ich nur einige bemerkenswerte Krankengeschichten auszugsweise anführen:

K. Gr., 42 Jahre alt. Aufnahme am 3. Juni 1910.

1894 Lues. Hg- und Jodkur. 1897 Rezidive, 4 Touren Hg. Dezember 1909 Abducensschwäche, durch Jodbehandlung gebessert. Mai 1910 Schwindelanfälle, halbseitige Parästhesien, Doppelbilder. Hg-Injektionen wurden nicht vertragen.

Status: Linke Pupille $<\mathrm{r}$., beide unausgiebig reagierend, linke Gliedmaßen schwächer, Tr.- und Per.R. 1. > r., P.S.R. $++1 .>$ r., Ach.S.R. $++1 .>$ r., Fußklonus links, Babinski l. + .

Parese des Rectus ext. und Obliqu. sup. oc. dextri. Wassermann +++ .

4. Juni. 0,4 Arsenobenzol in $20 \mathrm{~cm}$ alkalischer Lösung intramuskulär.

10. Juni entlassen. Keine Schwindel-oder Halbseitenerscheinungen. Doppelbilder nur mehr in extremer Blickrichtung.

25. Juni. Vollkommenes Wohlbefinden, kein Schwindel, keine Doppelbilder mehr.

15. Juli. Anhaltend beschwerdefrei. Gewichtszunahme, volle Leistungsfähigkeit. Pupillendifferenz geringer, Reaktion deutlich, aber wenig ausgiebig, A ugenmuskeln frei. Tiefe Reflexe lebhaft, l. Spur $>$ r., kein Klonus, kein Babinski.

31. August. Kontrolluntersuchung. Fühlt sich ganz gesund. Befund wie am 15. Juli. Wassermann ++ . Gewichtszunahme $4 \mathrm{~kg}$.

3. Oktober. Unverändert gut. Ach.S.R. l. $=$ r.

15. Februar. Keinerlei Beschwerden.

J. E., 27 Jahre alt. Aufnahme am 22. August 1910.

Januar 1910 lue tische Infektion. 45 Einreibungen mit Hg, Jod. 30. März Wassermann ++ . April Plaques an der Zunge, Papeln am Kopf. 5 Injektionen mit $\mathrm{Hg}$. salic. 11. Mai Wassermann -, geheilt, wegen Dyspepsie beurlaubt. Seit Fnde Juli Abnahme der Sehschärfe, Kopfschmerzen, vergeßlich, später A ufregungszustände, zeitweise, besonders nachts, verwirrt, aggressiv gegen die Umgebung, unde utliche S prache, ausgebreitete Geschwüre am Kopf und Nacken.

Status: Rechte Pupille $>1$, sehr träge reagierend, schlaffere Innervation 
des rechten Facialis. Sehnenreflexe gleich. Zahlreiche, teilweise exulcerierte Papeln im Bereiche der behaarten Kopfhaut und im Nacken.

Beiderseitige Stau ungspapille, erweiterte, geschlängelte Venen, vereinzelte, radiäre Hämorrhagien, Visus 6/18.

Dementes, apathisches Verhalten, beträchtliche Störung der Auffassung und Merkfähigkeit, verlangsamte, verwaschene Sprache. Gewicht $68 \mathrm{~kg}$.

25. August. 0,6 Arse nobenzol intramuskulär in alkalischer Lösung.

26. August. Nachts unruhig, desorientiert, bleibt nicht im Bett, verkennt die Umgebung.

27. August. Nachts unruhig, tagsüber freier, orientiert.

29. August. Papeln verkleinern sich, trocknen ein, Umgebung blässer. Ablehnendes Verhalten, gehemmt, faßt Fragen schwer auf.

3. September. Rasche Involution der papulösen Stellen. Psychisch frei, klar, fühlt sich viel wohler.

6. September. Psychisch keine Störung mehr nachweisbar, ohne Auffälligkeit. Geschwüre gänzlich abgeheilt. Kommt in die freie Abteilung. Augenspiegelbefund unverändert. Visus 6/12.

20. September. Vollkommenes Wohlbefinden. Gewichtszunahme $15 \mathrm{~kg}$.

28. September. Wassermann +++ !

5. Oktober. Geheilt entlassen.

E. E., 36 Jahre alt. Aufnahme am 5. September 1910.

Weiß nichts von luetischer Infektion. Am 14. August nach 12stündigem Ritt Parästhesien, Kälte- und Schwächegefühl im linken Bein, linksseitiger Gürtelschmerz. Rasch zunehmende Lähmung des linken Beines, Ameisenla ufen im rechten Bein, erschwerte Harnentleerung. Ende August schon deutliche Atro phie. Zu Hause 8 Einreibungen mit grauer Salbe ohne Erfolg.

Status: Linke Pupille stark verzogen, entrundet, $>$ r, beide etwas träge reagierend. Obere Extremitäten frei. Bd.R. nicht auslösbar. Cr.R. 0, P.S.R. l. < r., Ach.S.R. 1. $<$ r. 11. und 12. Brustwirbel druck- und klopfempfindlich, handbreite hyperästhetische Zone am Thorax links.

Beide Beine passiv frei beweglich. Aktive Bewegungen links kraftlos, unsicher, Muskulatur welk, stark atrophisch. Hyperästhesie von der linken Inguinalgegend nach abwärts. Erhebliche Störung der Tiefensensibilität. Im Bereiche des rechten Beines Analgesie und Thermanästhesie. Gang unmöglich.

7. September. Wassermann +++ .

12. September. 0,6 Arsenobenzol in $12 \mathrm{~cm}$ monacider Lösung intramuskulär.

15. September. Blasenentleerung normal. Bewegungen im Fußgelenk kräftiger. Muskeltonus besser. 20. September. Erhebliche Besserung der Motilität. P.S.R. l. > r. Ach.S.R. gleich. Hyperästhesie noch bestehend, rechts annähernd normale Sensibilität. Mittlerer und unterer Bd.R. links fehlend, Cr.R. links 0. Hyperästhetische Zone am Thorax kleiner. Deutlicher Rückgang der Atrophie.

2. Oktober. Beginnt zu gehen.

13. Oktober. Rasch fortschreitende Besserung. P.S.R. 1. > r., links leichter Patellarklonus. Sensibilitätsstörung an den Beinen geschwunden. Motorische Kraft sehr gut. Dumpfes Schmerzgefühl an der linken Brustseite noch vorhanden.

4. November. Pelziges Gefühl im linken Knie. Bewegungen im linken Sprunggelenk noch schwächer als rechts. Gang frei, kräftig. Linke Pupille $>$ r., wenig ausgiebig reagierend, rechte entrundet, besser reagierend. Unterer Bd.R. 
beiderseits 0, oberer Bd.R. l. fehlend, r. auslösbar. Cr.R. l. 0. P.S.R. I. > r., Ach.S.R. l. $>$ r. Keine Sensibilitätsstörung. Schmerz am Thorax unverändert.

17. November. Wassermann ++ . Einreibungskur mit $\mathrm{Hg}$ und Jod innerlich.

12. Dezember. Schmierkur beendet. Brustschmerz unbeeinflußt. Wassermann + .

16. Dezember. 0,7 Arsenobenzol in $12 \mathrm{~cm}$ monacider Lösung, intramuskulär.

12. Januar. Schmerzgefühl in der Brust geschwunden.

1. Februar. Wassermann -. 14. Februar. Vollkommenes Wohlbefinden, Pupillenbefund ohne Änderung, leichte Reflexdifferenz noch vorhanden. Gesund und diensttauglich.

15. März. Reitet stundenlang, steigt Berge, fühlt sich ganz gesund, macht mühelos und beschwerdefrei Truppendienst im Gebirgsterrain.

Au. W. Aufnahme am 17. November 1910.

Angeblich nur mehrmals weiche Geschwüre. Vor 2 Jahren ulceröse Papel am Scheitel und Kopfschmerzen. Nach Hg-Injektionskur und Jod Heilung. Jetzt seit 3 Wochen zunehmende Kopfschmerzen, seit 5 Tagen linksseitige Gesichtsnervenlähmung, Herabsetzung der Hörschärfe links, Schwerbeweglichkeit der Zunge nach rechts.

Status: Pupillen gleich, prompt reagierend. Ijinker Facialis in allen drei Ästen gelähmt. Hypoglossus links paretisch. Reflexe gesteigert. Hörschärfe nach Cerumenentfernung normal. mord.

16. November. Wassermann ++ . Droht wegen Kopfschmerz mit Selbst.-

18. November. 0,8 Arsenobenzol. Wegen rasender Kopfschmerzen Mo.

19. November. Nachts starke Kopfschmerzen.

20. November. Abnehmende Kopfschmerzen.

22. November. Fast sch merzfrei.

26. November. Kein Kopfschmerz, Augenschluß schon möglich.

30. November. Deutliche Innervation in allen Asten. Zunge frei beweglich.

12. Dezember. Nur mehr leichte Parese. Dauernd schmerzfrei.

4. Januar. Minimale Schwäche des linken Mundfacialis. Sonst vollkommen geheilt. Wassermann + .

19. Januar. Wohlbefinden, seit 14 Tagen voll diensttauglich.

1. Februar. Wassermann -

R. S., 31 Jahre alt. Aufnahme am 20. September 1910.

Vor 10 Jahren Lues, Hg- und Jodkur. Kein Rezidiv, keine Weiterbehandlung. Frühjahr 1910 Pupillendifferenz, $6 \mathrm{Hg}$-Injektionen, Jod. Seit 1 Monat intercostalneuralgische Schmerzen links, nach Jodbehandlung gebessert; seit 8 Tagen beiderseits konstante und heftige Intercostalschmerzen, zeitweise auch reißende Schmerzen in den Beinen.

Status: Rechte Pupille weit, reaktionslos, linke prompt. P.S.R. ++ gleich. Ach.S.R. ++ gleich. Wirbelsäule befundlos. Kein Sensibilitätsdefekt. Fundus normal.

23. September. Wassermann -.

10. Oktober. 0,6 Arsenobenzol.

14. Oktober. Keine Schmerzen mehr.

6. Dezember. Trotz körperlicher Anstrengung ganz beschwerdefrei. Gewichtszunahme. Pupillenbefund ohne Ãnderung. 
Die Erfolge der Salvarsanbehandlung bei Nervenkrankheiten.

15. Februar 1911. Fühlt sich, gesund wie ein Fisch", macht vollen Truppendienst.

R. St. Aufnahme am 20. November 1910.

1905 Ulcus, Hg.Kur. Nach 2 Monaten Iritis, Hg.Kur, Jod. Seit 2 Monaten Druckgefühl in Stirn und Hinterhaupt, seit 4 Wochen Schwindelanfälle mit Bewußtseinstrübung. 10. November. Wassermann ++ .

Status: Linke Pupille entrundet, exzentrisch, träge Reaktion. P.S.R. ++ Ach.S.R. ++ . Fundus bis auf leichte temporale Sichel normal.

22. November. 0,7 Arse nobenzol.

30. November. Kopfdruck und Schwindel geschwunden. Dienstbar entlassen.

11. Januar. Vollkommenes Wohlbefinden. Wassermann --.

Außerdem gehören in das Grenzgebiet zwischen echt syphilitischen und metaluetischen Erkrankungen 8 Fälle von Spätformen dubiosen Charakters. Bei diesen konnte nur in 2 Fällen ein bemerkenswerter Effekt bezüglich der nervösen Beschwerden und des Blutbefundes erzielt werden.

Tabelle I.

\begin{tabular}{|c|c|c|c|c|c|c|c|}
\hline Erkrankungsform & Zahl & $\begin{array}{c}\text { Heilung } \\
\text { bzw. } \\
\text { weit- } \\
\text { gehende } \\
\text { Besserung } \\
\end{array}$ & $\begin{array}{c}\text { Besse- } \\
\text { rung }\end{array}$ & $\begin{array}{c}\text { Vorïber- } \\
\text { gehende } \\
\text { Besse- } \\
\text { rung }\end{array}$ & $\begin{array}{l}\text { Ohne } \\
\text { Erfolg }\end{array}$ & $\begin{array}{c}\text { Vorüber- } \\
\text { gehende } \\
\text { Ver- } \\
\text { schlim- } \\
\text { merung }\end{array}$ & $?$ \\
\hline Latente Lues & $6+1$ & 6 & & & & 1 & \\
\hline Tertiäre Lues & 3 & 3 & & & & & \\
\hline Meningomyelitis & 1 & 1 & & & & & \\
\hline Lues spinalis & 1 & & 1 & & & & \\
\hline $\begin{array}{l}\text { Luet. Hemiplegie, } \\
\text { Endarteriitis }\end{array}$ & 7 & 3 & 1 & 1 & 1 & & 1 \\
\hline Lues cerebrospinalis & 2 & 1 & & & & & 1 \\
\hline Meningit. gummos. & 1 & 1 & & & & & \\
\hline Lues cerebralis & 4 & 1 & & 1 & 1 & & 1 \\
\hline Luet. Demenz & 1 & & & & 1 & & \\
\hline Luet. Neuralgie & 2 & & 1 & 1 & & & \\
\hline Pachymening. luet. & 1 & & & & 1 & & \\
\hline \multirow[t]{2}{*}{ Unklare Diagnose } & 1 & & & & & & 1 \\
\hline & 31 & 16 & 3 & 3 & 4 & 1 & 4 \\
\hline Dubiose Spätformen & 8 & 2 & 1 & 1 & 3 & & 1 \\
\hline
\end{tabular}

Hinsichtlich der Ergebnisse der Blutuntersuchung will ich bemerken, daß von den verwertbaren 25 Fällen obiger Gruppen 15 nach der Behandlung einen negativen Wassermann ergaben, davon 2 erst nach Wiederholung der Injektion, 3 erst nach 2-3 Monaten. Daß 
gerade bei den Spätformen Heileffekt und Blutbefund oft nicht paralle] gehen, will ich nur beiläufig hervorheben.

Tabelle II. Serologischer Blutbefund (Wassermann).

\begin{tabular}{|c|c|c|c|c|c|}
\hline Erkrankungsform & Zahl & $\begin{array}{l}\text { Vorher }+ \\
\text { nachher }-\end{array}$ & $\begin{array}{l}\text { Vorher }+ \\
\text { nachher } \\
\text { schwácher }\end{array}$ & $\begin{array}{l}\text { Vorher - } \\
\text { nachher - }\end{array}$ & $\begin{array}{c}\text { Vorher }+ \\
\text { nachher }+\end{array}$ \\
\hline Luetische Formen & 17 & 12 & 3 & & 1 \\
\hline \multirow[t]{2}{*}{ Dubiose Spätformen } & 8 & 3 & & 1 & 4 \\
\hline & 25 & 15 & 3 & 1 & 5 \\
\hline Tabes & 7 & 1 & \multirow{3}{*}{1} & \multirow[t]{3}{*}{3} & 3 \\
\hline Progr. Paralyse & 8 & & & & 7 \\
\hline $\begin{array}{l}\text { Verdacht auf progr. } \\
\text { Paralyse }\end{array}$ & 1 & & & & 1 \\
\hline & 16 & 1 & 1 & 3 & 11 \\
\hline
\end{tabular}

Die günstigen therapeutischen Erfolge der Salvarsanbehandlung bei wirklichen Spirochätenerkrankungen, sowie die von Alt auch an metaluetischen Erkrankungen erzielten Erfolge geben Veranlassung, daß man verhältnismäßig bald, trotz der ursprünglich von Ehrlich ausgesprochenen Warnung und trotz theoretisch vollkommen berechtigter Bedenken und begründeter Skepsis, auch Fälle von Tabes und Paralyse mit Arsenobenzol zu behandeln versuchte.

Für die Berechtigung dieser Versuche sprechen a priori mehrere Momente. Ich möchte nur darauf hinweisen, daß wir in vielen derartigen Fällen trotz gelegentlicher schlechter Erfahrungen immer wieder auf $\mathrm{Hg}$ und Jod greifen, wiewohl auch für diese Behandlung dasselbe Gegenargument der Unbehebbarkeit von Ausfällen degenerativer Natur gilt. Im übrigen wissen wir, daß speziell bei der Tabes, nebst den Symptomen, welche durch die degenerativen Veränderungen bedingt sind, auch Reizerscheinungen eine große Rolle spielen, die kaum anders als durch toxische Einwirkung oder durch Kombination mit entzündlich syphilitischen Meningealveränderungen hervorgerufen zu erklären sind. Solche Formen, die oft positiven Wassermann zeigen, sind es auch, welche bei antiluetischer Behandlung recht günstig reagieren. Des weiteren bedarf es keiner Erörterung, daß besonders Initialstadien mitunter ganz unlösbare differentialdiagnostische Schwierigkeiten gegen echt spätluetische Erkrankungsformen geben. Endlich stellt die organotonische und roborierende Nebenwirkung des Arsenobenzols einen gewiß nicht zu unterschätzenden Heilfaktor auch dort dar, wo sicher keine echte Spirochätenerkrankung mehr vorliegt. 
Von diesen Erwägungen ausgehend, haben zahlreiche Kliniker die Behandlungsversuche in Angriff genommen.

Die Schwierigkeit in der Einschätzung und Beurteilung von Behandlungserfolgen bei metaluetischen Formen, wo Spontanremissionen recht oft vorkommen, ist bekannt. In den oben angeführten Momenten, vielleicht auch in der Auswahl der Fälle, der angewandten Methode, sowie in den für den zu erwartenden Erfolg vorausgesetzten oder verlangten Kriterien dürften die divergierenden Urteile der verschiedenen Beobachter ihre Erklärung finden.

Jedenfalls haben die bisherigen Erfahrungen ergeben, daß Tabiker und Paralytiker, wenn Komplikationen fehlen, im allgemeinen die Salvarsanbehandlung gut vertragen und nur selten Verschlimmerungen vorkommen.

Hinsichtlich der Paralyse in ihren vorgeschrittenen Stadien sind alle Beobachter einig. Bei solchen Fällen ist nicht nur nichts zu erwarten, es ist im Gegenteil von Behandlungsversuchen mit Salvarsan abzuraten (Michaelis, H. Op penheim, Peritz, A. Pick, W. Pick, Plaut, Schlesinger usw.). Ebenso haben die Erfahrungen gezeigt, daß die Anwendung des Salvarsans bei Anfallsparalytikern jeden Stadiums schädlich und gefährlich ist, da in solchen Fällen rasche Verschlimmerungen, auch Todesfälle beobachtet werden konnten.

Fälle, für welche Alt die Bezeichnung vom „Wetterleuchten“ der Paralyse geschaffen hat, sind für die Beurteilung der Wirksamkeit irgendeiner Therapie so gut wie unverwertbar, weil dieses „Wetterleuchten" einen nicht zu fassenden Krankheitsbegriff darstellt. Bezüglich der initialen Formen hält die Mehrzahl der Autoren mit einem definitiven Urteil zurück. Willige spricht sich für die Möglichkeit aus, durch Salvarsan den Eintritt von Remissionen zu begünstigen. Fa user berichtet über Remissionen, die seiner Meinung nach nur als Effekt der Therapie anzusehen sind, Alt hält an der günstigen Beeinflussung speziell bei Taboparalysen fest. Ansonsten wird die Anwendung des Salvarsans bei beginnender Paralyse als gestatteter Versuch angesehen.

Meine eigenen Beobachtungen umfassen 16 sichere Paralysen, 2 Fälle fraglicher Diagnose (spätluetische Encephalitis oder pr. P.), 1 Fall, der vielleicht tatsächlich als „Wetterleuchten“ anzusprechen wäre, deshalb aber für die in Rede stehende Frage nicht verwertbar ist.

Ich konnte mich in keine $\mathrm{m}$ Falle, in welchem die Diagnose sicher oder auch nur wahrscheinlich war, von irgendeinem einwandfreien Erfolg überzeugen. 2 Fälle - ein Anfallsparalytiker und ein ausgesprochener Fall - erfuhren eine rasche Verschlimmerung.

Wesentlich günstiger liegen die Verhältnisse bezüglich der Aussichten einer Behandlung der Tabes. Für diese Erkrankung kommt 
in den Anfangsstadien und in gewissen Übergangsformen teils die spirillotrope, teils die organotrope Wirkung, bei manchen nur die roborierende Komponente des Salvarsans in Betracht. Die überwiegende Majorität der Beobachter berichtet tatsächlich über gute Erfolge. Diese Erfolge beziehen sich in erster Linie auf eine kürzer oder länger dauernde Beseitigung von Symptomen, die an sich spontane Schwankungen zeigen und als Reiz- oder Lähmungserscheinungen von den eigentlichen degenerativen Veränderungen des Nervensystems nicht direkt abhängig sind.

Es handelt sich um auffallende Besserung heftiger lanzinierender Schmerzen, Beseitigung von Krisen, günstige Beeinflussung der Ataxie (Alt, Bayet, Duhot, Loeb, Marschalko, Michaelis, Milian, Pal, Treupel, Werther u. a.).' Utber Besserung der Sensibilität berichtet Frenkel, Treupel, Wechselmann usw. Recht zahlreich sind auch die Beobachtungen, welche beweisen, daß das Salvarsan auch einen Einfluß auf objektive Symptome haben kann, wie es bei der bisherigen Behandlung seltener zu sein scheint. So konnten HeuckJaffé, Loeb, Marinesco, Marschalko, Nonne, Oppenheim, Peritz u. a. Besserung oder Wiederkehr der Pupillenreaktion, Ausgleich der Anisokorie, Marcus, Nonne, Michaelis Wiederkehr der Patellarsehnenreflexe und Achillessehnenreflexe, Marinesco und $0 p$ penheim rasche Heilung eines Mal perforant beobachten.

Als nicht unwesentlich möchte ich die zumeist eintretende Hebung des subjektiven Befindens, die Besserung des allgemeinen Kräftezustandes anführen, einen therapeutischen Effekt, welcher bei der Behandlung Tabeskranker doch auch von Bedeutung ist. Daß bei vorgeschrittenen Tabikern, mitunter auch bei initialen Fällen, meist vorübergehende Verschlimmerungen eintreten können, sowie daß in vielen Fällen keinerlei Erfolg beobachtet werden kann, ist ebenso sicher als die Tatsache, daß wir nach unseren klinischen Erfahrungen in keinem Falle mit Sicherheit Erfolg, Fehl- oder Mißerfolg vorausbestimmen können. Nach den publizierten Beobachtungen scheint aber, soweit aus diesen ein Urteil möglich ist, eine schädigende Wirkung bei unkomplizierten Tabesfällen doch nur selten, der günstige Einfluß häufiger.

Wenn ich über meine Erfahrungen berichten darf, so konnte ich bei den 12 Tabikern, die ich behandelt habe, fünf sehr günstige Erfolge beobachten, über welche ich in aller Kürze berichten will.

A. B., 58 Jahre alt. Aufnahme am 19. Dezember 1910.

1891 Lues. Zwei Schmierkuren. 1906 wegen Iritis Hg-Kur. 1907, 1908 Lipik Hg-Kur, am Schluß der Kur Intoxikationserscheinungen. 1909 wegen lanzinierender Schmerzen und nervöser Erscheinungen Kaltwasserkur.

September 1909 wegen Augenaffektion zwei Touren $\mathrm{Hg}$ und Jod.

Februar 1910 angeblich Iritis, Hg-Kur, Jod. Jetzt zeitweise heftige Sch merzen. 
Status: Linke Pupille $>$ r., linke entrundet, beide unausgiebig reagierend. De utlicher Romberg. P.S.R. 0, Ach.S.R. 0, PI.R. lebhaft. Biernacki +. Abadie -. Wassermann -.

20. Dezember. 0,6 Salvarsan.

25. Januar. Wohlbefinden, keine Schmerzen, Rhomberg sehr gering. Sonst objektiv unverändert.

10. Februar. Keine Änderung. Wassermann -.

E. Chr., 32 Jahre alt. Aufnahme am 30. Juni 1910.

Vor 12 Jahren infiziert. Kein Exanthem, kein Rezidiv, keine Behandlung. 1900 Pupillendifferenz, seit 1905 zunehmende Ataxie, häufige intensive, lanzinierende Sch merzen, Inkontinenzerscheinungen seitens der Blase. 1909 Drïsengeschwulst an der linken Halsseite. Jod und Arsen ohne Erfolg. Juni 1910 Wassermann ++ .

Status: Rechte Pupille $>$ l., beide lichtstarr. P.S.R. 0, Ach.S.R. 0, Rhomberg + , hochgradige Ataxie, Biernacki + , Abadie + , Bulbi auf Druck unempfindlich. Ausges prochene Störungen der taktilen und Tiefensensi bilität. Gänseeigroßer Drüsentumor am Halse. Gewicht $54 \mathrm{~kg}$.

Fundus normal. Innere Organe ohne wesentlichen Befund.

7. Juli. Intramuskuläre Injektion $0,4: 20 \mathrm{ccm}$ in alkalischer Lösung.

20. Juli. Zunehmende Besserung des Allgemeinbefindens, viel kräftiger. Blasenstörung behoben.

15. August. Sensibilität objektiv nachweisbar gebessert. Ataxie geringer. Gehvermögen besser, weniger Ermüdung.

12. September. Nachuntersuchung ergibt Bestand der subjektiven und objektiven Besserung. Drüsengeschwulst kleinhühnereigroß. Gewicht $58 \mathrm{~kg}$. Sonstige objektive Symptome unverändert. Nur selten leich te lanzinierende Schmerzen.

14. Dezember. Wassermann -, sonst gleich.

20. Januar 1911. Besserung anhaltend, Patient nahezu schmerzfrei.

Al. Gr., 43 Jahre alt. Aufnahme am 5. September 1910.

1899 Lu^s. Hg-Kur. Kein Rezidiv. 1901 und 1902 ohne direkten AnlaB in Lipik je eine Hg-Einreibungskur und Jod. Seit Monaten hä ufig la nzi nierende Sch merzen, Kopfdruck, häufige Lar ynxkrisen, körperlich herabgekommen, nervöse Angstgefühle.

Status: Pupillen annähernd gleich, nicht ganz prompt auf Licht reagierend. P.S.R. gleich, schwach auslösbar, Ach.S.R. 0, Rhomberg +, Bulbus rechts unterempfindlich auf Druck. Biernacki + , Abadie + . Mäßige Ataxie. Wassermann +++ . Gewicht $61 \mathrm{~kg}$.

5. September. 0,6 Arse nobenzol in $12 \mathrm{ccm}$ monacider Lösung.

29. September. Fühlt sich,,wie neugeboren“, kein Kopfschmerz, kein Angstgefühl, trotz Bewegung fast schmerzfrei. Seit 14 Tagen keine Larynx krise. P.S.R. schwach r. $>$ 1., Ach.S.R. 0, sonst unveränderter objektiver Befund. Gewicht $68 \mathrm{~kg}$.

17. Oktober. Fast beschwerdefrei. Kein Hus te na nfall, Gang ohne Störung.

26. Oktober. Wassermann +++ .

11. November. Gutes Befinden anhaltend, Keine Krisen.

17. Januar 1911. Fühlt sich gesund. Macht Truppendienst ohne alle Beschwerden.

29. Januar. Wohlbefinden.

G. L., 56 Jahre alt. Aufnahme am 23. Januar 1911.

Vor 25 Jahren Lues, Hg-Kur und Lipik. Kein Rezidiv. Später noch zwei 
Hg-Kuren und Jod, zuletzt 1906. Seither jährlich 8 Wochen Jod. Seit $10 \mathrm{Jah}$ ren lanzinierende Schmerzen, Ermüdbarkeit, Unsicherheit beim Gehen, gastrische Krisen, Gürtelgefühl. Schmerzen in letzter Zeit sehr häufig. 7. Januar. Wassermann -.

Status: Beide Pupillen eng, verzogen, nur eine Spur auf Licht reagierend. Bulbi unterempfindlich auf Druck. Biernacki +. P.S.R. r. schwach, l. 0; Ach.S.R. 1. 0 , r. schwach. Rhomberg + , mäßige Ataxie. Fundus normal.

25. Januar. 0,6 Salversan.

12. Februar. Allgemeinbefinden sehr gut, anhaltend schmerzfrei, Gang sicherer, kräftiger, kein Gürtelgefühl.

1. März. Dauernd frei von lanzinierenden Schmerzen, keine Krise.

4 Fälle wurden nur vorübergehend gebessert, 3 blieben, abgesehen von subjektiver Besserung, unbeeinflußt, kein einziger wurde durch die Behandlung verschlimmert.

Bevor ich das Gesamtergebnis über den heutigen Stand unserer Erfahrung aufstellen will, möchte ich nur noch einige Worte über Methode, Dosierung und die Frage der Reinjektion sagen.

Aus den Erfahrungen, welche bei der Behandlung der frischen Syphilis gewonnen wurden, haben sich alle Suspensionen wegen Unzuverläßlichkeit, Langsamkeit der Wirkung, schlechter Dauererfolge, starker Infiltrate und Neigung zu Hautnekrosen nicht bewährt. Ebenso sind stark alkalische, sowie stark saure Lösungen wegen der großen Schmerzhaftigkeit und der starken toxischen Wirkung nicht empfehlenswert.

Es kommen also eigentlich nur die intravenösen Applikationen der alkalischen Lösung und die intramuskuläre, schwach alkalische bzw. schwach saure klare Lösung in Betracht. Die intravenöse Injektion an sich ist wohl frei von wesentlichen Nachteilen, reicht aber bei einmaliger Anwendung infolge der raschen Ausscheidung für einen vollen Effekt nicht aus, weshalb die Wiederholung oder die Unterstützung durch Setzung eines intramuskulären Depots in Anwendung gebracht werden muß.

In neurologischen Fällen handelt es sich aber nicht darum, eine möglichst rasche, gewaltige Einwirkung auf massenhafte, im Blutstrom kreisende Spirochäten, sondern um spirochätenarme Krankheitsprozesse, bei welchen die Spirochäten, wo sie überhaupt vorhanden sind, überdies in Zellen und Geweben schwer zugänglich deponiert sind, weshalb eine gleichsam sterilisierende Durchspülung ihren Zweck nicht erreicht.

Dazu kommt, daß wir recht oft mit Fällen zu tun haben, bei welchen die intravenöse Applikation wegen der damit verbundenen Alteration des Blutdruckes und der Herzaktion die größte Vorsicht geboten ist.

Ich halte daher für die neurologische Praxis trotz aller Mängel, welche der intramuskulären Anwendung von Lösungen anhaften (Nekrose in der Tiefe, Infiltration, unkontrollierbare Resorption), diese Methode als die derzeit empfehlenswertere. 
Sie verbindet die wünschenswerte Raschheit der Wirkung mit den Vorteilen der Dauerwirkung und läßt gerade die organotrope Komponente, die in unseren Fällen nicht unwesentlich ist, in höhere $m$ Maße zur Geltung kommen. Was die Dosis anlangt, so scheinen doch die bisherigen Behandlungen dafür zu sprechen, daß große Dosen nicht besser wirken als mittlere, daß auch die Dauerwirkung nicht proportional der Größe der Einzeldosis ist (Lesser, Weintraud). Über die Wirksamkeit und eventuelle Gefahren einer wiederholten Anwendung der Salvarsanbehandlung - intermittierende Depotbehandlung, sowie über die von Lesser, Kromayer, jüngst auch von Lazarus empfohlene fraktionierte Behandlung mit mehrfachen kleinen Dosen - ist derzeit ein Urteil noch nicht möglich. Jedenfalls ist aber in Fällen, welche sich gegen eine mittlere Dosis refraktär verhalten haben, eine Reinjektion zwecklos. Ist nach intramuskulärer Applikation die Wirkung nicht ausreichend, dann würde ich raten, nicht vor Ablauf von 2 Monaten eine Wiederholung der Injektion zu versuchen, sondern lieber vorher eine Hg-Kur einzuschalten. Dies um so mehr, als zahlreiche Beobachtungen dafür sprechen, $\mathrm{daB}$ sich die Salvarsanbehandlung in vorteilhafter Weise mit $\mathbf{H g}$ - und Jodkuren kombinieren läßt und recht häufig kombiniert zur Anwendung gelangen muß.

Am Schlusse meiner Ausführungen angelangt, möchte ich das Hauptergebnis in folgenden Sätzen zusammenfassen:

Das Salvarsan ist bei einwandfreier Technik und zweckmäßiger Methode ein nahezu ungefährliches, für echt syphilitische Erkrankungen des Nervensystems der tertiären Syphilisperiode ungemein energisches und günstig wirksames Heilmittel. Hinsichtlich der Raschheit des zu erreichenden Effektes erweist es sich dem $\mathrm{Hg}$ und Jod überlegen. Es ist daher dessen Anwendung bei frischen Fällen cerebraler und spinaler Erkrankungen, wo es auf eine besonders rasche Wirkung ankommt, direkt indiziert, ebenso in Fällen, bei welchen $\mathrm{Hg}$ versagt oder unanwendbar ist. A uch bei Tabes, besonders bei den aktiven Formen, gibt die Salvarsanbehandlung häufig sehr gute Erfolge hinsichtlich der Reizerscheinungen.

Bei unkomplizierten beginnenden Paralysen ist ein Behandlungsversuch erlaubt, bei ausgesprochenen Fällen ist keine Wirkung zu erwarten. Als relative Kontraindikation, d. h. als Kontraindikation gegen die intravenöse Anwendung, kann die Komplikation mit Herzfehlern, schweren Herzneurosen, stärkerer Arteriosklerose, sowie mit höhergradigem Alkoholismus und Diabetes gelten. Absolut kontraindiziert ist die Salvarsananwendung überhaupt nach Vorbehandlung mit Atoxyl, Enesol u. dgl., bei Nerven- 
krankheiten mit Lokalisation in wichtigen Zentren, bei größeren Hirnherden, speziell bei Tumorformen, bei sehr folgeschweren degenerativen Erkrankungen, besonders bei vorgeschrittener Paralyse und bei allen Anfallsparalytikern.

\section{Literaturverzeichnis.}

Alt, Das neueste Ehrlichsche Präparat gegen Syphilis. Münch. med. Wochenschr. 1910, Nr. 11.

Derselbe, Über ein neues Arsenpräparatzur Behandlung der Syphilis und Metasy philis. Münch. med. Wochenschr. 1910, Nr. 31.

Derselbe, Sitzungsbericht. Deutsche med. Wochenschr. 1910, Nr. 41, 46. Basch, Sitzungsbericht. Med. Klin. 1911, Nr. 1.

Ba yet, L'Arse nobenzol. Journ. de Bruxelles 1910, No. 41 ; Le scalpel et Liège med. 1910, No. 21 ; ref. Münch. med. Wochensch. 1910, 2588.

Beck, Über transitorische Fasererkrankungen des N. vestibul. bei mit Ehrlich - Hata 606 behandelten Kranken. Med. Klin. 1910, 1969.

Benario, Über syphilitische Neurorezidive. Münch. med. Wochenschr. 1911, Nr. 1.

Blaschko, Kritische Bemerkungen zur Ehrlich-Hata-Behandlung. Berl. klin. Wochenschr. 1910, Nr. 35.

Brasch, Sitzungsbericht. Münch. med. Wochenschr. 1911, 281.

Bonhöffer, Sitzungsbericht. Berl. klin. Wochenschr. 1910, H. 35.

Bruhns, Zur Frage der Therapie mit 606. Berl. klin. Wochenschr. 1910, H. 50.

Chrzelitzer, Meine Erfahrungen mit Ehrlich - Hata 606. Münch. med. Wochenschr. 1910, H. 48.

Citron, Sitzungsbericht. Deutsche med. Wochenschr. 1910, 2272.

Dobrovits, Erfahrungen über 606. Wiener med. Wochenschr. 1910, H. 40.

Doerr, Erfahrungen mit dem Ehrlichschen Mittel. Wiener klin. Wochenschr. 1910, 987.

Derselbe, Sitzungsbericht. Wiener klin. Wochenschr. 1910, H. 50 .

Donagh, Mc., Syphilisbehandlung mit Ehrlich - Hata 606. Ref. Wiener klin. Wochenschr. 1910, 1537; Münch. med. Wochenschr. 1910, H. 46.

Duhot, Syphilisbehandlung mit Ehrlich 606. Annales de la Policl. de Bruxelles 1910; ref. Münch. med. Wochenschr. 1910, H. 39 und Ehrlich. Abhandlungen über Salvarsan. München 1911.

Ehrlich, P., Sitzungsbericht. Deutsche med. Wochenschr. 1910, H. 41.

Derselbe, Nervenstörungen und Salvarsanbehandlung. Berl. klin. Wochenschr. 1910, H. 51.

Derselbe, Pro und contra Salvarsan. Wiener med. Wochenschr. 1911, H. 1.

Derselbe, Die Salvarsantherapie. Rückblicke und Ausblicke. Münch. med. Wochenschr. 1911, H. 1.

Derselbe, Abhandlungen über Salvarsan. München 1911.

Derselbe, Anwendung und Wirkung des Salvarsans. Deutsche med. Wochenschr. 1910, H. 52.

Ehlers, Ein Todesfall nach Ehrlich 606. Münch. med. Wochenschr. 1910, H. 42.

Eversbusch, Über eine schwere Nebenwirkung des Salvarsans. Münch. med. Wochenschr. 1911, H. 7. 
Faure, Salvarsa nbehandlung der Tabes. Ref. Münch. med. Wochenschr. $1911,547$.

Fauser, Einige Mitteilungen über das Resultat von 118 Einspritzungen mit den Ehrlichschen Arsen präparaten. Württemb. med. Korr.Bl. $1910, \mathrm{H}, 43$.

Favento, Ưber 156 mit Ehrlich - Hata behandelten Fälle. Münch. med. Wochenschr. 1910, H. 40.

Derselbe, Weitere Erfahrungen über 256 mit Ehrlich - Hata 606 behandelte Fälle. Zit. nach Ehrlichs Abhandlungen, München 1911.

Finger, Die Behandlung der Syphilis mit Ehrlichs Arsenobenzol. Wiener klin. Wochenschr. 1910, H. 47.

Fleckseder, Sitzungsbericht. Med. Klin. 1910, H. 46.

Fraenkel, Sitzungsbericht. Deutsche med. Wochenschr. 1911, 187.

Fraenkel-Grouven, Erfahrungen mit dem Ehrlichschen Mittel 606. Münch. med. Wochenschr. 1910, H. 34.

Frenkel, Die Anwendung des Ehrlich-Hata-Mittels bei Nerven. krankheiten. Berl. klin. Wochenschr. 1910, H. 45.

Friedländer, Sitzungsbericht. Deutsche med. Wochenschr. 1910, H. 41.

Fordyce, Further observ. on the use of the Ehrlich - Hata-Prep. 606. New York med. Journ. 1910, H. 19, 20; ref. Münch. med. Wochenschr. 1910, $2502,2559$.

Gaucher, Gazette des hôpitaux 1910, H. 30.

Gennerich, Zur Technik und Kontraindikation der Salvarsan. behandlung. Münch. med. Wochenschr. 1910, H. 52.

Geronne, Umfrage. Med. Klin. 1910, H. 40.

Derselbe, Die intravenöse Therapie der Syphilis mit Ehrlich - Hata 606. Berl. klin. Wochenschr. 1910, H. 49.

Geronne-Guttmann, Zur Frage der Neurotropie des Salvarsans. Berl. klin. Wochenschr. 1911, H. 11.

Glück, Kurzer Bericht über 109 mit 606 behandelten Luesfälle. Münch. med. Wochenschr. 1910, H. 31 .

Grósz, Arsenobenzol gegen syphilitische Augenleiden. Deutsche med. Wochenschr. 1910, H. 37, 50.

Hamel, Sitzungsbericht. Deutsche med. Wochenschr. 1911, H. 3, S. 142.

Herxheimer-Schonnefeld, Weitere Mitteilungen über die Wirkung des Ehrlichschen Arsenobenzols bei Syphilis. Med. Klin. 1910, H. 36.

Heuck-Jaffé, Weitere Mitteilungen über das Ehrlichsche Dioxydiamidoarsenobenzol. Deutsche med. Wochenschr. 1911, H. 6.

Hirsch, Ehrlichbehandlung bei luetischen Augenerkrankungen. Münch. med. Wochenschr. 1910, H. 49.

Hügel-Ruete, Unsere bisherigen Erfahrungen mit dem EhrlichHataschen Arsen präparat 606. Münch. med. Wochenschr. 1910, H. 39.

Igersheimer, Ex perimentelle und klinische Untersuchungen mit dem Dioxydiamidoarsenobenzol. Münch. med. Wochenschr. 1910, H. 51.

Intosh, Mc., u. Fildes, The Theory and Practice of the Treatment of Syph. with Ehrlich New Specific. The Lancet, Dez. 1910; ref. Münch. med. Wochenschr. 1911, H. 1.

Isaac, Sitzungsbericht. Deutsche med. Wochenschr. 1911, H. 2.

Ivanyi, Meine Erfahrungen mit Ehrlich 606. Wiener med. Wochenschr. 1910, H. 36, 45.

Jadassohn, Unsere Erfahrungen mit 606. Deutsche med. Wochenschr. 1910 , H. 51. 
Jorgensen, Ein Fall tödlicher Arsenvergiftung bei Behandlung von Gehirnsyphilis mit Ehrlich 606. Med. Klin. 1911, H. 10.

Kopp, Erfahrungen bei Behandlung mit Salvarsan. Münch. med. Wochenschr. 1911, H. 5.

Korach, Sitzungsbericht. Deutsche med. Wochenschr. 1911, 235.

Korczynski, Therapeutische Erfahrungen mit dem Ehrlich-Hata. schen Arsen präparat. Österr. Ärzte-Ztg. 1910, H. 19, 20.

Kromayer, Die chronische Syphilisbehandlung mit 606 nach Erfahrungen an 400 Fällen. Deutsche med. Wochenschr. 1910, H. 49.

Lazarus, Sitzungsbericht. Deutsche med. Wochenschr. 1911, 185.

Ledermann, Ưber Erfahrungen mit Ehrlich 606 bei subcutaner und intramuskulärer Anwendung. Med. Klin. 1910, H. 50.

Lesser, Zur Wirkungs- und Anwendungsweise von Salvarsan. Berl. klin. Wochenschr. 1911, H. 4.

Loeb, Über 130 mit Ehrlich - Hata 606 behandelte Fälle. Ärztl. Mitt. a. u. f. Baden 1910, H. 20.

Derselbe, Erfahrungen mit Ehrlich 606. Münch. med. Wochenschr. 1910, H. 30.

Derselbe, Weitere Erfahrungen über 606. Zit. nach Ehrlichs Abhandlungen, München 1911.

Marcus, Die Salvarsanbehandlung bei sy philitischen Erkrankungen des Nervensystems. Münch. med. Wochenschr. 1911, H. 2.

Marinesco, Sur quelques résultats obtenues par le „606“dans le Traitement des Maladies nerveuses. La Présse Méd. 1911, H. 8.

Marschalko, Ưber die ungenügende Dauerwirkung der neutralen Suspension von Salvarsan bei Syphilis. Deutsche med. Wochenschr. 1911, H. 5 .

Mattauschek, Sitzungsbericht. Wiener med. Wochenschr. 1910, 2832.

Derselbe, Sitzungsbericht. Wiener klin. Wochenschr. 1910, 1825.

Matzenauer, Sitzungsbericht. Wiener klin. Wochenschr. 1910, H. 48.

Mayer, Max, Erfahrungen mit dem Ehrlich - Hata-Präparat 606 bei metaluetischen Erkrankungen. Deutsche med. Wochenschr. 1910, 1832.

Meidner, Ehrlichs Syphilisheilmittel bei innerer Lues. Therap.d. Gegenw. 1910, H. 9.

Meirowsky, Die Einwirkung des Ehrlichschen Mittels a uf den syphi. litischen Proze B. Med. Klin. 1910, H. 42.

Michaelis, 110 Fälle von Syphilisbehandlung nach Ehrlich-Hata. Berl. klin. Wochenschr. 1910, H. 37.

Derselbe, Die Ehrlichbehandlung in der internen Medizin. Deutsche med. Wochenschr. 1910, H. 49.

Milian, Le „606“. Progr. Méd. 1910, H. 43.

Derselbe, Sitzungsbericht. Münch. med. Wochenschr. 1910, 2502.

Neisser, Die Behandlung der Syphilis mit dem Ehrlichschen Präparat 606. Deutsche med. Wochenschr. 1910, H. 41.

Neisser - Kuznitzky, Über die Bedeutung des Ehrlichschen Arsenobenzols für die S y philis behandlung. Berl. klin. Wochenschr. 1910, H. 32.

Nicholsen - Fordyce, Journ. of the Amer. med. Assoc. 1910; ref. Neurol. Centralbl. 1910, 1311.

Nonne, Sitzungsbericht. Deutsche med. Wochenschr. 1911, 141.

Oppenheim, H., Meine Erfahrungen mit Ehrlich-Hata-Behandlung bei syphilogenen Nervenkrankheiten. Deutsche med. Wochenschr. 1910, H. 49. 
Oppenheim, M., Sitzungsbericht. Wiener med. Wochenschr. 1910, 2956.

Pal, 606 bei Tabes. Wiener klin. Wochenschr. 1910, H. 26.

Derselbe, Sitzungsbericht. Wiener klin. Wochenschr. 1910, H. 50.

Peritz, Sitzungsbericht. Deutsche med. Wochenschr. 1911, 91.

Pick, A., Sitzungsbericht. Deutsche med. Wochenschr. 1910, H. 51.

Pick, W., Bericht über die bisherigen Resultate der Behandlung der Syphilis mit den Präparaten von Ehrlich-Hata. Wiener klin. Wochenschr. 1910, H. 33.

Plaut, Sitzungs bericht. Münch. med. Wochenschr. 1911, 282.

Plehn, Sitzungsbericht. Deutsche med. Wochenschr. 1911, 89.

Pötz]-Schüller, Über letale Hirnschwellung bei Syphilis. Zeitschr. f. d. ges. Neur. u. Psych. 3, H. 1, 2. 1910.

Riecke, Über das Ehrlichsche Syphilisheilmittel 606. Deutsche med. Wochenschr. 1911, 243.

Derselbe, Fortschr. d. Medizin 1910, H. 49.

Riehl, Über Syphilisbehandlung mit Ehrlichschem Heilmittel. Wiener klin. Wochenschr. 1910, H. 45.

Rille, Über eventuelle Nebenwirkungen an den Hirnnerven bei Behandlung mit Ehrlichschem Präparat 606. Berl. klin. Wochenschr. 1910, H. 50.

Ritter, Unsere Erfahrungen mit 606. Münch. med. Wochenschr. 1910, 43.

Rosenthal, Über 606. Berl. klin. Wochenschr. 1910, H. 47.

Rumpel, Unsere bisherigen Erfahrungen mit dem Ehrlichschen Präparat 606. Deutsche med. Wochenschr. 1910, H. 49.

Derselbe, Sitzungsbericht. Med. Klin. 1911, H. 10.

Sänger, Sitzungsbericht. Deutsche med. Wochenschr. 1911, 142.

Salmon, Sitzungsbericht. Deutsche med. Wochenschr. 1910, H. 41.

Sieskind, Zusammenfassender Bericht über 375 mit Ehrlich - Hata behandelte Fälle. Münch. med. Wochenschr. 1910, H. 39.

Spiethoff, Arsenobenzol bei Syphilis. Münch. med. Wochenschr. 1910, H. 35 .

Derselbe, Salvarsan bei Syphilis. Münch. med. Wochenschr. 1911, H. 4.

Schanz, Das Ehrlich - Präparat bei Augenkrankheiten. Münch. med. Wochenschr. 1910, H. 45.

Derselbe, Salvarsanbehandlung und Neur. opt. Münch. med. Wochenschr. 1911, H. 10.

Schlesinger, Erfahrungen über das Ehrlich-Hata-Präparat in internen und neurologischen Fällen. Wiener med. Wochenschr. 1910, H. 46.

Scholz-Salzberger-Beck, Über die Behandlung der Syphilis mit Arsenobenzol. Deutsche med. Wochenschr. 1910, H. 50.

Stark, Die Behandlung der Syphilis mit Ehrlich - Hata 606. Ärztl. Mitt. a. u. f. Baden 1910, H. 21.

Stern, Meine Erfahrungen mit Ehrlich-Hata 606. Deutsche med. Wochenschr. 1910, H. 49.

Taege, Behandlung der Sy philis mit Ehrlich - Hata 606. Münch. med. Wochenschr. 1910, H. 42, 45.

Tomaczewski, Zusammenfassende Übersicht der Salvarsanbehandlung der Syphilis. Beiheft d. Med. Klin. 1911, H. 1.

Treupel, Erfahrungen und Erwägungen mit dem neuen EhrlichHataschen Mittel bei syphilitischen und metasy philitischen Er. krankungen. Deutsche med. Wochenschr. 1910, H. 30. 
716 E. Mattauschek: Die Erfolge der Salvarsanbehandlung bei Nervenkrankheiten.

Derselbe, Weitere Erfahrungen mit Ehrlich - Hata-Injektionen, insbesondere bei Lues des Zentralnervensystems, bei Tabes und Paralyse. Münch. med. Wochenschr. 1910, H. 46.

Treupel-Levi, Die klinische Prüfung des Dioxydiamidoarsenobenzols. Münch. med. Wochenschr. 1911, H. 5, 6.

Ullmann, Zur Ehrlichschen Therapie mit Arsenobenzol. Wiener med. Wochenschr. 1910, H. 49.

Derselbe, Sitzungsbericht. Wiener klin. Wochenschr. 1910, H. 49.

Volhard, Ärtl. Mitt. a. u. f. Baden 1910, H. 20.

Weber, Sitzungsbericht. Münch. med. Wochenschr. 1911, H. I.

Wechselmann, Behandlung der Syphilis mit Dioxydiamidoarsenobenzols. Berlin 1910.

Derselbe, Beobachtungen an 503 mit Dioxydiamidoarsenobenzol behandelte Krankheitsfälle. Deutsche med. Wochenschr. 1910, H. 32.

Weintraud, Erfahrungen mit dem Ehrlich - Hataschen Syphilisheilmittel 606. Med. Klin. 1910, H. 43.

Werther, Meine Erfahrungen mit 606. Münch. med. Wochenschr. 1910, H. 48.

Derselbe, Über die Neurorezidiven nach Salvarsan. Münch. med. Wochenschr. 1911, H. 10.

Willige, Über Erfahrungen mit Ehrlich - Hata 606 an psychiatrischneurologischem Materiale. Münch. med. Wochenschr. 1910, H. 46.

Zeissl, Bericht über die Behandlung der ersten 100 Fälle mit Ehrlich 606. Wiener med. Wochenschr. 1910, H. 34, 38.

Derselbe, Einige Bemerkungen zur Behandlung mit Salvarsan. Wiener med. Wochenschr. 1911, H. 4.

Zieler, Erfahrungen mit Ehrlich-Hata 606. Deutsche med. Wochenschr. 1910, H. 44. 